\title{
ON KERNELS OF SOME LEFT RESTRICTION SEMIGROUPS IN $\wp_{X}$
}

\author{
${ }^{1}$ Abubakar, R. B.; ${ }^{2}$ Asibong-ibe, U.I. \& ${ }^{3}$ Jackreece, P.C. \\ ${ }^{1,2,3}$ Department of Mathematics and Statistics, \\ University of Port Harcourt, \\ Rivers state, Nigeria \\ Email: rafiatabubakar86@gmail.com
}

\begin{abstract}
This study presents some left restriction semigroups LRS in partial transformation $\wp \boldsymbol{I}_{\{\mathbf{3 , 4}, \mathbf{5 , 6 , 7 , 8 , 9 , 1 0 \}}}$ and computed the kernels $K\left(\wp \mathfrak{I}_{\{\mathbf{3 , 4}, \mathbf{5 , 6 , 7 , 8 , 9 , 1 0}\}}\right)$ for each element of LRS in $\wp \mathfrak{I}_{X}$ •
\end{abstract}

Key words: Kernels, Partial transformation, ideals, Left Restriction semigroups.

\section{INTRODUCTION}

Restriction semigroups are semigroups with an additional unary operation ${ }^{+}$(left restriction) and * (right restriction). A restriction semigroup is a bi-unary semigroup which is both left and right restriction which also satisfies the linking identities

$$
\left(a^{+}\right)^{*}=a^{+} \text {and }\left(a^{*}\right)^{+}=a^{*}
$$

A semigroup $\mathrm{S}$ is left restriction with respect to $E \subseteq E(S)$ if `

i. $\quad E$ is a subsemilattice of $\mathrm{S}$

ii. Every element $a \in S$ is $\widetilde{R_{E}}$-related to an element of $E$ (denoted by $a^{+}$)

iii. $\widetilde{R_{E}}$ is a left congruence

iv. The left ample condition holds $\forall a \in S$ and $e \in E$,

$$
a e=(a e)^{+} a
$$

Equivalently,

A semigroup $S$ is a right restriction with respect to $E=E(S)$ if

i. $\quad E$ is a subsemilattice of $\mathrm{S}$

ii. Every element $a \in S$ is $\widetilde{L_{E}}$-related to an element of $E$ (denoted by $a^{*}$ )

iii. $\widetilde{L_{E}}$ is a right congruence

iv. The right ample condition holds $\forall a \in S$ and $e \in E$,

$$
e a=a(e a)^{*}
$$


Hollings (2009) opined that the most natural way to represent restriction semigroup was through partial transformation $\wp \mathfrak{I}_{X}$. Abubakar, Asibong-ibe and Jackreece (2020) computed some LRS embedded in partial transformation $\wp \widetilde{I}_{X}$ and enumerated the congruence classes $\widetilde{R_{S}}$ and the semilattices of idempotents $\widetilde{E_{S}}$ of the LRS for $\wp \widetilde{\Im}_{\{2,3,4,5,6,7,8,9,10\}}$. Algebra studies sets endowed with operations that generate interesting structures on them. For elements $a$ and $b$ of a semigroup $\mathrm{S}$, Green relations, $\mathcal{L}, \mathcal{R}, \mathfrak{J}, \mathcal{H}, D$, are defined by

$$
\begin{gathered}
a \mathcal{L} b \text { iff } S a=S b \\
a \mathcal{R} b \text { iff } a S=b S \\
a \mathfrak{I} b \text { iff } S a S=S b S \\
a \mathcal{H} b \text { iff } a \mathcal{L} b \text { and } a \mathcal{R} b
\end{gathered}
$$

$a D b$ iff there exists a $c$ in $S$ such that $a \mathcal{L} c$ and $c \mathcal{R} b$

That is, a and $\mathrm{b}$ are $\mathcal{L}$-related if they generate the same left ideal, $\mathcal{R}$-related if they generate the same right ideal and $\mathfrak{J}$-related if they generate the same two-sided ideal. These are equivalence relations on $\mathrm{S}$, so each of them yields a partition of $S$ into equivalence classes . Kernels is an important property in algebra that defines quotient objects which also measures injectivity. In computer, windowsNT has a Hybrid kernel which is monolithic where all services run in kernel mode or a macro kernel where services run in user mode. Kernels communicates with hardware and manages resources .Kernel forms a bridge between applications and actual data to be processed. For a finite semigroups $S$, there is a unique minimal ideal called the kernel of $S, K(S)$. $\Im(a)$ is the kernel (Howie, 2004). Pertrich and Rankin (1992) observed that every congruence on an inverse semigroup is completely determined by its idempotent and these were what was referred to as kernel normal systems. Consider

$$
f: G \rightarrow H
$$

The kernel of $f$ is a set of elements of $\mathrm{G}$ which map into the identity element $e \in G$. Kernel of

$$
f=\{a \in G: f(a)=e\}
$$

For a group morphism, the kernel of $f$, denoted by $\operatorname{ker} f$ is the set of elements of $G$ that are mapped by $f$ to the identity of $\mathrm{H}$. That is,

$$
\text { Ker } f=\left\{g \in G: f(g)=e_{H}\right\}
$$

The kernels of a semigroup $S$ are the set of elements mapped to the same elements (Howie, 2003). Hollings and Gould (2010) further defined Left restriction semigroups as algebra defined by the following identities:

$$
\begin{array}{ll}
\text { i. } & (x y) z=x(y z) \\
\text { ii. } & x^{+} x=x \\
\text { iii. } & x^{+} y^{+}=y^{+} x^{+} \\
\text {iv. } & \left(x^{+} y\right)=x^{+} y^{+} \\
\text {v. } & x y^{+}=(x y)^{+} x
\end{array}
$$

Let $\mathrm{S}$ be a semigroup, if $\mathrm{S}$ is simultaneously isomorphic to a subsemigroup of a partial transformation $\wp T_{X}$ that is closed under ${ }^{+}$and to a subsemigroup of some $\wp T_{X}$ that is closed 
under * and if in addition the images of the semilattices $S^{+}$and $S^{*}$ coincides, then we call $\mathrm{S}$ a two sided restriction semigroup with respect to $S^{+}=S^{*}$.

Let $\wp T_{X}$ be a left restriction with distinguished semilattice

And with

$$
E=\left\{I_{Y}: Y \subseteq X\right\}
$$

$$
\alpha^{+}=1_{\text {dom } \alpha}
$$

$\mathrm{S}$ is left restriction if and only it embeds in some $\wp T_{X}$ in a way that preserves ${ }^{+}$where

$$
\alpha^{+}=\alpha \alpha^{-1} \quad \text { (Hollings, 2009) }
$$

If $\mathrm{S}$ is a subsemigroup of some $\wp \widetilde{I}_{X}$, and $\mathrm{S}$ is closed under ${ }^{+}$, i.e. if $S^{+} \subseteq S$, then, we call $\mathrm{S}$ a left restriction semigroup with respect to $S^{+}$(Hollings, 2009). Considering $\mathrm{T}$ as a subsemigroup of some $\wp \mathfrak{I}_{X}^{*}$, we put

$$
T^{*}=\left\{\alpha^{*}: \alpha \in T\right\} \subseteq E(T) .
$$

Also, if we let $\mathrm{T}$ be a subsemigroup of some $\wp \mathfrak{J}^{*}{ }_{X}$, if $\mathrm{T}$ is closed under $*$, i.e. if $T^{*} \subseteq T$, then we call $\mathrm{T}$ a right restriction semigroup with respect to $T^{*}$ (Hollings, 2009). Let $\mathrm{S}$ be left adequate, for $a \in S$, let $a^{+}$denote the unique idempotent in the $R^{*}-$ class of a (as it coincides with the unique idempotent in the $\tilde{R}$ - class of a). If $S$ satisfies the left ample condition that for all $a \in S$ and $e \in E(S)$

$$
a e=(a e)^{+} a
$$

Then $\mathrm{S}$ is a left ample ( formerly left type A ) semigroup (Hollings and Gould, 2010) . Left restriction semigroup has been treated as the idempotent connected Ehresmann semigroup who was drawing connections between semigroup theory and category-theoretic work of Ehresmann with the goal of applying techniques from category theory to semigroup theory. Equivalently, $\mathrm{S}$ is a Left Restriction Semigroup

Let $\mathrm{S}$ be an inverse semigroup, then with $E=E(S)$

- $E$ is a semilattce

- $\widetilde{R_{E}}=R$ is a left congruence

- Every $R$ - class contains an idempotent, we have

$$
a^{+}=a a^{\prime}
$$

- For any $a \in S$ and $e \in E$

$$
(a e)^{+} a=(a e)(a e)^{\prime} a=a e\left(e a^{\prime}\right) a=a e\left(a^{\prime} a\right)=a\left(a^{\prime} a\right) e=a e
$$

Hence, $\mathrm{S}$ is left restriction with respect to $E(S)$; dually, $\mathrm{S}$ is a right restriction, so that $\mathrm{S}$ is restriction [6] . In this paper, we compute the kernels of some LRS in $\wp \Im_{X}$,

$K\left(\wp \mathfrak{I}_{\{3,4,5,6,7,8,9,10\}}\right)$. 


\section{MAIN RESULT}

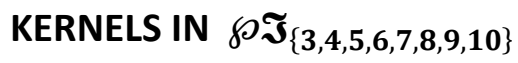

i. $\quad \wp T_{\{1,2,3\}}$ given by

$$
\begin{aligned}
& S_{1}=\left\{A, B, C, D, F, G, H, I, J, K, L, M, N, O, P, Q, R, S, T, U, V, W X, Y, A_{1}, B_{1}, C_{1}, E\right\} \\
& \mathrm{A}=\left(\begin{array}{lll}
1 & 2 & 3 \\
1 & x & 2
\end{array}\right), B=\left(\begin{array}{lll}
1 & 2 & 3 \\
1 & x & x
\end{array}\right), C=\left(\begin{array}{lll}
1 & 2 & 3 \\
x & 2 & x
\end{array}\right) D=\left(\begin{array}{lll}
1 & 2 & 3 \\
x & x & 2
\end{array}\right), F=\left(\begin{array}{lll}
1 & 2 & 3 \\
3 & x & x
\end{array}\right), \\
& G=\left(\begin{array}{lll}
1 & 2 & 3 \\
2 & x & x
\end{array}\right), H=\left(\begin{array}{lll}
1 & 2 & 3 \\
x & 1 & x
\end{array}\right), I=\left(\begin{array}{lll}
1 & 2 & 3 \\
x & x & 1
\end{array}\right), \mathrm{J}=\left(\begin{array}{lll}
1 & 2 & 3 \\
x & 3 & x
\end{array}\right), K=\left(\begin{array}{lll}
1 & 2 & 3 \\
x & x & 3
\end{array}\right), \\
& L=\left(\begin{array}{lll}
1 & 2 & 3 \\
3 & 2 & x
\end{array}\right), M=\left(\begin{array}{lll}
1 & 2 & 3 \\
3 & x & 2
\end{array}\right), N=\left(\begin{array}{lll}
1 & 2 & 3 \\
2 & 3 & x
\end{array}\right), O=\left(\begin{array}{lll}
1 & 2 & 3 \\
2 & x & 3
\end{array}\right), \mathrm{P}=\left(\begin{array}{lll}
1 & 2 & 3 \\
1 & 3 & x
\end{array}\right), \\
& Q=\left(\begin{array}{lll}
1 & 2 & 3 \\
1 & x & 3
\end{array}\right), R=\left(\begin{array}{lll}
1 & 2 & 3 \\
x & 2 & 3
\end{array}\right), S^{\prime}=\left(\begin{array}{lll}
1 & 2 & 3 \\
x & 3 & 2
\end{array}\right), T=\left(\begin{array}{lll}
1 & 2 & 3 \\
x & 1 & 2
\end{array}\right), U=\left(\begin{array}{lll}
1 & 2 & 3 \\
x & 2 & 1
\end{array}\right), \\
& V=\left(\begin{array}{lll}
1 & 2 & 3 \\
2 & x & 1
\end{array}\right), W=\left(\begin{array}{lll}
1 & 2 & 3 \\
2 & 1 & x
\end{array}\right), \quad X=\left(\begin{array}{lll}
1 & 2 & 3 \\
1 & 2 & x
\end{array}\right), Y=\left(\begin{array}{lll}
1 & 2 & 3 \\
3 & 1 & x
\end{array}\right), A_{1}=\left(\begin{array}{lll}
1 & 2 & 3 \\
3 & x & 1
\end{array}\right) \\
& B_{1}=\left(\begin{array}{lll}
1 & 2 & 3 \\
x & 3 & 1
\end{array}\right), C_{1}=\left(\begin{array}{lll}
1 & 2 & 3 \\
x & 1 & 3
\end{array}\right), \varepsilon=\left(\begin{array}{lll}
1 & 2 & 3 \\
x & x & x
\end{array}\right)
\end{aligned}
$$


Table $1: \wp T_{\{1,2,3\}}:\left(\begin{array}{lll}1 & x & 2\end{array}\right)$

\begin{tabular}{|c|c|c|c|c|c|c|c|c|c|c|c|c|c|c|c|c|c|c|c|c|c|c|c|c|c|c|c|c|}
\hline & A & 1 & & & $\mathrm{~F}$ & $G$ & $\mathrm{H}$ & I & $\mathbf{J}$ & $\mathrm{K}$ & $\mathrm{L}$ & $\mathrm{M}$ & $\mathrm{N}$ & $\mathrm{O}$ & $P$ & Q & $\mathrm{R}$ & $S$ & $\mathrm{~T}$ & $\mathrm{U}$ & V & $\mathrm{W}$ & $x$ & $\mathrm{Y}$ & $A_{1}$ & $B_{1}$ & $C_{1}$ & \\
\hline A & B & B & D & $\mathrm{E}$ & $\mathrm{F}$ & $\mathrm{G}$ & I & & $\mathrm{K}$ & A & $\mathrm{M}$ & $\mathrm{F}$ & $\mathrm{O}$ & $G$ & Q & B & $\mathrm{D}$ & K & & $\mathrm{D}$ & $\mathrm{G}$ & V & $\mathrm{A}$ & & $\mathrm{F}$ & K & I & $F$ \\
\hline B & B & B & $\mathrm{P}$ & & $\mathrm{F}$ & $\mathrm{G}$ & $E$ & $\mathrm{E}$ & $\mathrm{F}$ & B & $\mathrm{F}$ & $\mathrm{F}$ & $\mathrm{G}$ & $\mathrm{G}$ & $\mathrm{P}$ & $\mathrm{P}$ & $\mathrm{P}$ & $\mathrm{F}$ & $\mathrm{E}$ & $\Gamma$ & $\mathrm{G}$ & $\mathrm{G}$ & B & & $\mathrm{F}$ & & E & $\mathrm{F}$ \\
\hline & $\mathrm{E}$ & & & & & & $\mathrm{H}$ & & & & & & & 1 & & & & & & & & & & $\mathrm{H}$ & & & $\overline{\mathrm{H}}$ & $\mathrm{I}$ \\
\hline D & $\mathrm{E}$ & & & & & I & I & & & D & $\mathrm{D}$ & $\mathrm{E}$ & & $\mathrm{E}$ & & & & & & & & & & & & & I & E \\
\hline F & $\bar{G}$ & & & 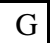 & & $\mathrm{E}$ & $E$ & $\mathrm{~F}$ & $\mathrm{E}$ & $\mathrm{F}$ & $\mathrm{E}$ & $\mathrm{C}$ & $\mathrm{E}$ & $F$ & F & & & G & & & & & $F$ & $E$ & & & $\mathrm{~F}$ & $\mathrm{E}$ \\
\hline G & l & & & & & & B & & & & & & & & & & & & & & & & $\mathrm{G}$ & B & & & B & \\
\hline $\mathrm{H}$ & & & & & & ( & $\mathrm{E}$ & & & $\mathrm{H}$ & & & $\mathrm{C}$ & C & & & & & & & & & $\mathrm{H}$ & $\mathrm{J}$ & & & E & E \\
\hline I & 1 & & & & & $\mathrm{D}$ & $\mathrm{E}$ & 1 & $\mathrm{t}$ & 1 & $\mathrm{H}$ & $\mathrm{K}$ & D & D & 1 & & & $\mathrm{E}$ & & & & $\mathrm{D}$ & I & $\mathrm{K}$ & & & $\mathrm{E}$ & E \\
\hline . & $c$ & & . & & $\mathrm{E}$ & E & $\mathrm{E}$ & & $\mathrm{E}$ & $\mathrm{J}$ & $E$ & C & $\mathrm{E}$ & $\mathrm{J}$ & 1 & & & & 8 & & & E & & E & 1 & & $\mathrm{~J}$ & E \\
\hline K & $\mathrm{D}$ & $\mathrm{K}$ & $\bar{K}$ & I & & $\mathrm{E}$ & $\bar{E}$ & . & $\mathrm{H}$ & $K$ & $\mathrm{E}$ & $\mathrm{D}$ & $\mathrm{E}$ & $\mathrm{K}$ & $\mathrm{E}$ & $\mathrm{K}$ & $\mathrm{K}$ & $\mathrm{D}$ & $\mathrm{D}$ & & & $\mathrm{F}$ & $\mathrm{K}$ & E & & & $\mathrm{K}$ & E \\
\hline $\mathrm{L}$ & $\mathrm{C}$ & & & & & $\mathrm{F}$ & $\mathrm{H}$ & & & $\mathrm{F}$ & C & $\mathrm{G}$ & $\mathrm{J}$ & $\mathrm{F}$ & $\mathrm{J}$ & & $\mathrm{L}$ & iv & $\mathrm{W}$ & $X$ & & $\mathrm{H}$ & $\mathrm{C}$ & $\mathrm{H}$ & & & $Y$ & $\mathrm{E}$ \\
\hline $\mathrm{M}$ & & & & & & E & I & & & $\mathrm{F}$ & & $\mathrm{G}$ & $\mathrm{K}$ & $\mathrm{F}$ & $\mathrm{K}$ & & $\mathrm{M}$ & $\Omega$ & $\mathrm{V}$ & & & & & & & & $\mu_{1}$ & \\
\hline $\mathrm{N}$ & C & $\Lambda$ & & & & E & B & $\mathrm{H}$ & $\mathrm{E}$ & $\mathrm{J}$ & $\mathrm{G}$ & C & D & $\mathrm{J}$ & $\mathrm{F}$ & & $\mathrm{N}$ & $\mathrm{L}$ & $X$ & 1 & $\mathrm{H}$ & B & G & $B$ & 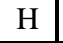 & $\mathrm{Y}$ & $P$ & $E$ \\
\hline $\mathrm{O}$ & $\mathrm{D}$ & $\mathrm{O}$ & & & & $\mathrm{E}$ & $\mathrm{B}$ & & & $\mathrm{K}$ & G & $\mathrm{D}$ & $\mathrm{F}$ & $\mathrm{K}$ & $\mathrm{F}$ & $\mathrm{K}$ & $\mathrm{O}$ & $\mathrm{M}$ & $\mathrm{A}$ & $\mathrm{V}$ & I & $\mathrm{B}$ & $\mathrm{G}$ & B & 1 & $A_{1}$ & $\mathrm{Q}$ & $E$ \\
\hline $\mathrm{P}$ & $X$ & $\mathrm{~B}$ & & ( & & $\mathrm{G}$ & $E$ & 1 & & & $\mathrm{H}$ & 1 & 0 & $\mathrm{~N}$ & $\mathrm{~F}$ & & & $\mathrm{C}$ & $C$ & 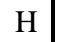 & $\mathrm{W}$ & $\mathrm{G}$ & B & $\mathrm{F}$ & & $\mathrm{H}$ & $\mathrm{J}$ & $\mathrm{E}$ \\
\hline $\mathrm{Q}$ & A & & $C$ & & & $\mathrm{G}$ & $\mathrm{E}$ & I & $t$ & $\mathrm{~K}$ & $\mathrm{~F}$ & $\mathrm{M}$ & $\mathrm{G}$ & 0 & B & $Q$ & $\mathrm{~K}$ & $\mathrm{D}$ & D & & $\mathrm{V}$ & $G$ & B & $F$ & $A_{1}$ & 1 & $\mathrm{~K}$ & $L$ \\
\hline $\mathrm{R}$ & $\mathrm{D}$ & & $\mathrm{C}$ & $\mathrm{D}$ & $\mathrm{E}$ & $\mathrm{E}$ & $\mathrm{H}$ & I & $\mathrm{J}$ & $\mathrm{K}$ & C & $\mathrm{D}$ & $\mathrm{I}$ & $\mathrm{K}$ & $\mathrm{J}$ & $\mathrm{r}$ & $\mathrm{R}$ & $\mathrm{S}$ & $\mathrm{T}$ & $\mathrm{U}$ & I & $\mathrm{H}$ & C & $\mathrm{H}$ & I & $B_{1}$ & $C_{1}$ & $E$ \\
\hline$S$ & $\mathrm{C}$ & & D & & & $\mathrm{E}$ & I & & $k$ & $\mathrm{~J}$ & I & $C$ & $\mathrm{~K}$ & $\mathrm{~J}$ & $\mathrm{H}$ & & & $\mathrm{R}$ & $\mathrm{U}$ & & $\mathrm{H}$ & & $\mathrm{D}$ & I & $\mathrm{H}$ & 1 & $B_{1}$ & $\mathrm{E}$ \\
\hline $\mathrm{T}$ & $\mathrm{H}$ & & & $\mathrm{F}$ & & $\mathrm{C}$ & I & E & K & $\mathrm{T}$ & $\mathrm{S}$ & $\mathrm{J}$ & $\mathrm{R}$ & $\mathrm{C}$ & $C_{1}$ & $\mathrm{~F}$ & D & $\mathrm{K}$ & 1 & $\mathrm{D}$ & $\mathrm{C}$ & $\mathrm{U}$ & $\mathrm{T}$ & $B_{1}$ & J & $\mathrm{K}$ & I & E \\
\hline $\mathrm{U}$ & I & $\mathrm{I}$ & $c$ & & & D & $\mathrm{H}$ & E & $\mathrm{J}$ & $\mathrm{U}$ & $\mathrm{R}$ & $\mathrm{K}$ & 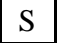 & D & $B_{1}$ & & C & & $\mathrm{H}$ & $\mathrm{C}$ & $\mathrm{D}$ & $\mathrm{T}$ & U & 1 & $\mathrm{~K}$ & $\mathbf{J}$ & $\mathrm{H}$ & E \\
\hline V & I & 1 & $\mathrm{C}$ & $\mathrm{F}$ & $\mathrm{K}$ & $\mathrm{D}$ & $\mathrm{B}$ & E & - & $\nabla$ & $\mathrm{O}$ & $\mathrm{K}$ & $\mathrm{M}$ & $\mathrm{D}$ & $A_{1}$ & 1 & $\mathrm{G}$ & $\mathrm{F}$ & B & $\mathrm{G}$ & $\mathrm{D}$ & $\mathrm{A}$ & $\mathrm{V}$ & $\mathrm{Q}$ & $\mathrm{K}$ & $\mathrm{F}$ & B & $\mathrm{E}$ \\
\hline W & $\mathrm{H}$ & $\mathrm{H}$ & $c$ & $\mathrm{H}$ & $\mathrm{J}$ & $\mathrm{C}$ & B & E & $\mathrm{F}$ & $\mathrm{W}$ & $\mathrm{N}$ & $\mathrm{JI}$ & $\mathrm{L}$ & $\mathrm{C}$ & $\mathrm{Y}$ & $\mathrm{H}$ & $\mathrm{G}$ & $\mathrm{F}$ & B & $\mathrm{G}$ & $\mathrm{C}$ & $\mathrm{X}$ & $\mathrm{W}$ & $\mathrm{P}$ & $\mathrm{J}$ & $\mathrm{F}$ & $\mathrm{B}$ & $\mathrm{E}$ \\
\hline$X$ & 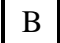 & $\mathrm{F}$ & & & & $G$ & $\mathrm{H}$ & E & J & $X$ & L & \begin{tabular}{|l} 
\\
\end{tabular} & 11 & 0 & 1 & 1 & C & & $\mathrm{H}$ & C & $\mathrm{G}$ & $\mathrm{W}$ & $\mathrm{X}$ & $\mathrm{Y}$ & $\mathrm{F}$ & $\mathrm{J}$ & $\mathrm{H}$ & $\mathrm{E}$ \\
\hline Y & $\mathrm{W}$ & $\mathrm{H}$ & $Y$ & $C$ & $\mathrm{~J}$ & C & $\mathrm{E}$ & B & E & $\mathrm{F}$ & $\mathrm{J}$ & $\mathrm{N}$ & C & $\mathrm{L}$ & $\mathrm{H}$ & $\mathrm{Y}$ & $\mathrm{F}$ & $\mathrm{G}$ & $\mathrm{G}$ & B & $X$ & $\mathrm{C}$ & $\mathrm{H}$ & $\mathrm{J}$ & $P$ & B & $\mathrm{F}$ & $\mathrm{E}$ \\
\hline$A_{1}$ & V & I & $A$ & G & $\mathrm{K}$ & $\mathrm{D}$ & $\mathrm{E}$ & B & E & $\mathrm{F}$ & $\mathrm{K}$ & $\mathrm{O}$ & $\mathrm{D}$ & $\mathrm{M}$ & I & $A_{1}$ & $\mathrm{~F}$ & $\mathrm{G}$ & $\mathrm{G}$ & $\mathrm{B}$ & $\mathrm{A}$ & $\mathrm{D}$ & I & $\mathrm{K}$ & $\mathrm{Q}$ & B & $\mathrm{F}$ & E \\
\hline$B_{1}$ & $\mathrm{U}$ & I & \begin{tabular}{|l|}
$D_{1}$ \\
\end{tabular} & C & $\mathrm{K}$ & $\mathrm{D}$ & E & $\mathrm{H}$ & $E$ & $\mathrm{~J}$ & $\mathrm{~K}$ & $\mathrm{R}$ & D & $\mathrm{S}$ & I & $B_{1}$ & . & $\mathrm{C}$ & $\mathrm{C}$ & $\mathrm{H}$ & $\mathrm{T}$ & $\mathrm{D}$ & I & $\mathrm{K}$ & $C_{1}$ & $\mathrm{H}$ & $\mathrm{J}$ & E \\
\hline 4 & $\mathrm{~T}$ & $\mathrm{H}$ & $C_{1}$ & D & $\mathrm{J}$ & $\mathrm{C}$ & E & 1 & E & K & $\mathrm{J}$ & $\mathrm{S}$ & $\mathrm{C}$ & $\mathrm{R}$ & $\mathrm{H}$ & $C_{1}$ & K & $\mathrm{D}$ & $\mathrm{D}$ & I & $\mathrm{U}$ & $\mathrm{C}$ & $\mathrm{H}$ & $\mathrm{J}$ & $B_{1}$ & I & $\mathrm{K}$ & E \\
\hline $\mathrm{E}$ & E & E & $\mathrm{E}$ & E & 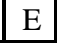 & $E$ & $E$ & $\mathrm{E}$ & E & 5 & E & E & $E$ & $E$ & E & $\mathrm{E}$ & E & $\mathrm{E}$ & $E$ & $E$ & $\mathrm{E}$ & $\mathrm{E}$ & $\mathrm{E}$ & $E$ & $\mathrm{E}$ & $\mathrm{E}$ & $\mathrm{E}$ & \\
\hline
\end{tabular}




$$
\begin{aligned}
& K_{A}=\{A, B\}_{B} ;\{C, D, G\}_{E} ;\{L, M\}_{G} ;\{J, N\}_{C} ;\{K, O, R\}_{D} \\
& K_{B}=\{A, B, P, Q\}_{B} ;\left\{T, H, W, Y, C_{1}\right\}_{H} ;\left\{U, V, A_{1}, B_{1}\right\}_{I} \\
& K_{C}=\{A, D, M, S, T\}_{D} ;\{N, O, G, V, W\}_{G} ;\{C, L, R, U, X\}_{C} \\
& K_{D}=\{A, B, C, D, G, H, I, T, U, V, W, X, E\}_{E} ;\left\{F, L, M, Y, A_{1}\right\}_{G} ;\left\{K, O, Q, C_{1},\right\}_{D} ;\left\{J, N, P, S, B_{1}\right\}_{C} \\
& K_{F}=\{A, B, P, Q, X\}_{F} ;\{C, D, F, M, N, O, G, R, S, E, J, K, L\}_{E} ;\left\{H, T, W, Y, C_{1}\right\}_{J} ;\left\{I, U, V, A_{1}, B_{1}\right\}_{K} \\
& K_{G}=\{A, B, P, Q, X\}_{G} ;\{C, D, F, G, J, K, L, M, N, O, R, S, E\}_{E} ;\left\{I, U, V, A_{1}, B_{1}\right\}_{D} ;\left\{H, W, Y, C_{1}\right\}_{C} \\
& K_{H}=\left\{A, B, F, H, I, J, K, P, Q, Y, A_{1}, B_{1}, C_{1}, E\right\}_{E} ;\{G, N, O, V, W\}_{B} ;\{A, D, M, S, T\}_{I} \\
& K_{I}=\{A, B, C, D, G, H, I, T, U, V, W, X, E\}_{E} ;\left\{F, L, M, Y, A_{1}\right\}_{B}, ;\left\{J, N, P, S, B_{1}\right\}_{H}, ;\{K, O, Q, R\}_{I} \\
& K_{J}=\left\{B, H, I, J, K, P, Q, Y, A_{1}, B_{1}, C_{1}, E\right\}_{E} ;\{A, D, M, S, T\}_{K} ;\{C, L, R, U, X\}_{J} ;\{G, N, O, V, W\}_{F} \\
& K_{K}=\left\{F, L, M, Y, A_{1}\right\}_{F} ;\{K, O, Q, R\}_{K} ;\left\{J, N, P, S, B_{1}\right\}_{J} \\
& K_{L}=\{F, J, K, E\}_{E} ;\{G, N, O\}_{C} ;\left\{I, A_{1}, B_{1}\right\}_{K} ;\{G, N, O\}_{G} \\
& K_{M}=\{A, B, X\}_{F} ;\{F, L, M\}_{G} ;\{K, O, R\}_{D} ;\{I, T, W\}_{J} \\
& K_{N}=\{F, J, K, E\}_{E} ;\{H, Y\}_{C} ;\left\{I, A_{1}, B_{1}\right\}_{D} ;\{B, P, Q\}_{G} ;\{G, N, O\}_{F} ;\{C, L, R\}_{J} \\
& K_{O}=\{F, L, M\}_{F} ;\{A, B, X\}_{G} ;\{C, D, G\}_{E} ;\{I, U, V\}_{D} \\
& K_{P}=\{F, N, O\}_{F} ;\{F, J, K\}_{E} ;\{B, P, Q\}_{B} ;\left\{H, Y, C_{1}\right\}_{H} \\
& K_{Q}=\{A, B\}_{B} ;\{F, L, M\}_{F} ;\{K, O, R\}_{K} ;\{I, U, V\}_{I} \\
& K_{R}=\{A, D\}_{D} ;\left\{J, P, B_{1}\right\}_{J} ;\{G, V, W\}_{G} ;\left\{F, Y, A_{1}\right\}_{F} \\
& K_{S}=\{A, D, T\}_{K} ;\{H, B, I, E\}_{E} ;\left\{K, Q, C_{1}\right\}_{D} ;\{C, U, X\}_{J} ;\left\{F, Y, A_{1}\right\}_{G} ;\left\{P, I, A_{1}\right\}_{C} \\
& K_{T}=\{A, D, T\}_{I} ;\{H, B, I, E\}_{E} ;\left\{K, Q, C_{1}\right\}_{D} ;\{G, V, W\}_{B} ;\left\{F, Y, A_{1}\right\}_{G} \\
& K_{U}=\{A, D, T\}_{I} ;\{H, B, I, E\}_{E} ;\left\{J, P, B_{1}\right\}_{H} ;\left\{K, Q, C_{1}\right\}_{I} ;\{G, V, W\}_{B} ;\left\{F, Y, A_{1}\right\}_{G} \\
& K_{V}=\{A, B, X\}_{G} ;\{C, D, G, E\}_{E} ;\{J, N, S\}_{H} ;\{F, L, M\}_{B} ;\{K, O, R\}_{I} ;\{I, U, V\}_{D} \\
& K_{W}=\{J, K, E\}_{E} ;\{G, N, O\}_{B} ;\{B, P, Q\}_{G} ;\{C, L, R\}_{H} ;\left\{I, A, B_{1}\right\}_{D} ;\left\{H, Y, C_{1}\right\}_{C} \\
& K_{X}=\{C, L, R\}_{C} ;\{D, M, S\}_{D} ;\{G, N, O\}_{G} ;\{B, P, Q\}_{B} ;\left\{A_{1}, B_{1}, I\right\}_{I} ;\{H, Y\}_{H} \\
& K_{Y}=\{F, P, Q\}_{E} ;\{C, L, R\}_{H} ;\{G, N, O\}_{B} ;\{B, P, Q\}_{F} ;\left\{A_{1}, B_{1}, I\right\}_{K} \\
& K_{A_{1}}=\{A, B, X\}_{F} ;\{C, D, G\}_{E} ;\{F, L, M\}_{B} ;\{J, T\}_{H} ;\{I, U, V\}_{K} ;\{H, T, W\}_{J} \\
& K_{B_{1}}=\{A, D, T\}_{K} ;\{B, H, I, E\}_{E} ;\left\{J, P, B_{1}\right\}_{H} ;\{G, V, W\}_{F} ;\left\{F, Y, A_{1}\right\}_{B} \\
& K_{C_{1}}=\left\{J, P, B_{1}\right\}_{J} ;\{B, H, I, E\}_{E} ;\{C, U, X\}_{H} ;\{G, V, W\}_{B} ;\left\{F, Y, A_{1}\right\}_{F} \\
& K_{E}=\left\{A, B, H, C, D, E, F, G, H, I, J, K, L, M, N, O, P, Q, R, S, T, U, V, W, X, Y, A_{1}, B_{1}, C_{1}\right\}_{E}
\end{aligned}
$$




$$
\begin{aligned}
& \text { ii. } P T_{\{1,2,3,4\}} \\
& S_{2}=\{A, B, C, D, F, G, H, I, J, K, L, M, N, O, P, E\} \text { where } \\
& A=\left(\begin{array}{llll}
1 & 2 & 3 & 4 \\
1 & 4 & x & x
\end{array}\right), B=\left(\begin{array}{llll}
1 & 2 & 3 & 4 \\
1 & x & x & x
\end{array}\right), C=\left(\begin{array}{llll}
1 & 2 & 3 & 4 \\
x & 3 & x & x
\end{array}\right), D=\left(\begin{array}{llll}
1 & 2 & 3 & 4 \\
4 & x & x & x
\end{array}\right), F=\left(\begin{array}{llll}
1 & 2 & 3 & 4 \\
3 & x & x & x
\end{array}\right), \\
& G=\left(\begin{array}{llll}
1 & 2 & 3 & 4 \\
2 & x & x & x
\end{array}\right), H=\left(\begin{array}{llll}
1 & 2 & 3 & 4 \\
x & 1 & x & x
\end{array}\right) I=\left(\begin{array}{llll}
1 & 2 & 3 & 4 \\
x & 4 & x & x
\end{array}\right), J=\left(\begin{array}{llll}
1 & 2 & 3 & 4 \\
x & x & 4 & x
\end{array}\right), K=\left(\begin{array}{llll}
1 & 2 & 3 & 4 \\
x & x & x & 4
\end{array}\right), \\
& L=\left(\begin{array}{llll}
1 & 2 & 3 & 4 \\
3 & 4 & x & x
\end{array}\right), M=\left(\begin{array}{llll}
1 & 2 & 3 & 4 \\
x & 2 & x & x
\end{array}\right), N=\left(\begin{array}{llll}
1 & 2 & 3 & 4 \\
4 & 3 & x & x
\end{array}\right), O=\left(\begin{array}{llll}
1 & 2 & 3 & 4 \\
x & x & 3 & x
\end{array}\right), P=\left(\begin{array}{llll}
1 & 2 & 3 & 4 \\
3 & x & 4 & x
\end{array}\right), \\
& \varepsilon=\left(\begin{array}{llll}
1 & 2 & 3 & 4 \\
x & x & x & x
\end{array}\right)
\end{aligned}
$$

Table 2 : $\wp T_{\{1,2,3,4,\}}:(14 x x)$

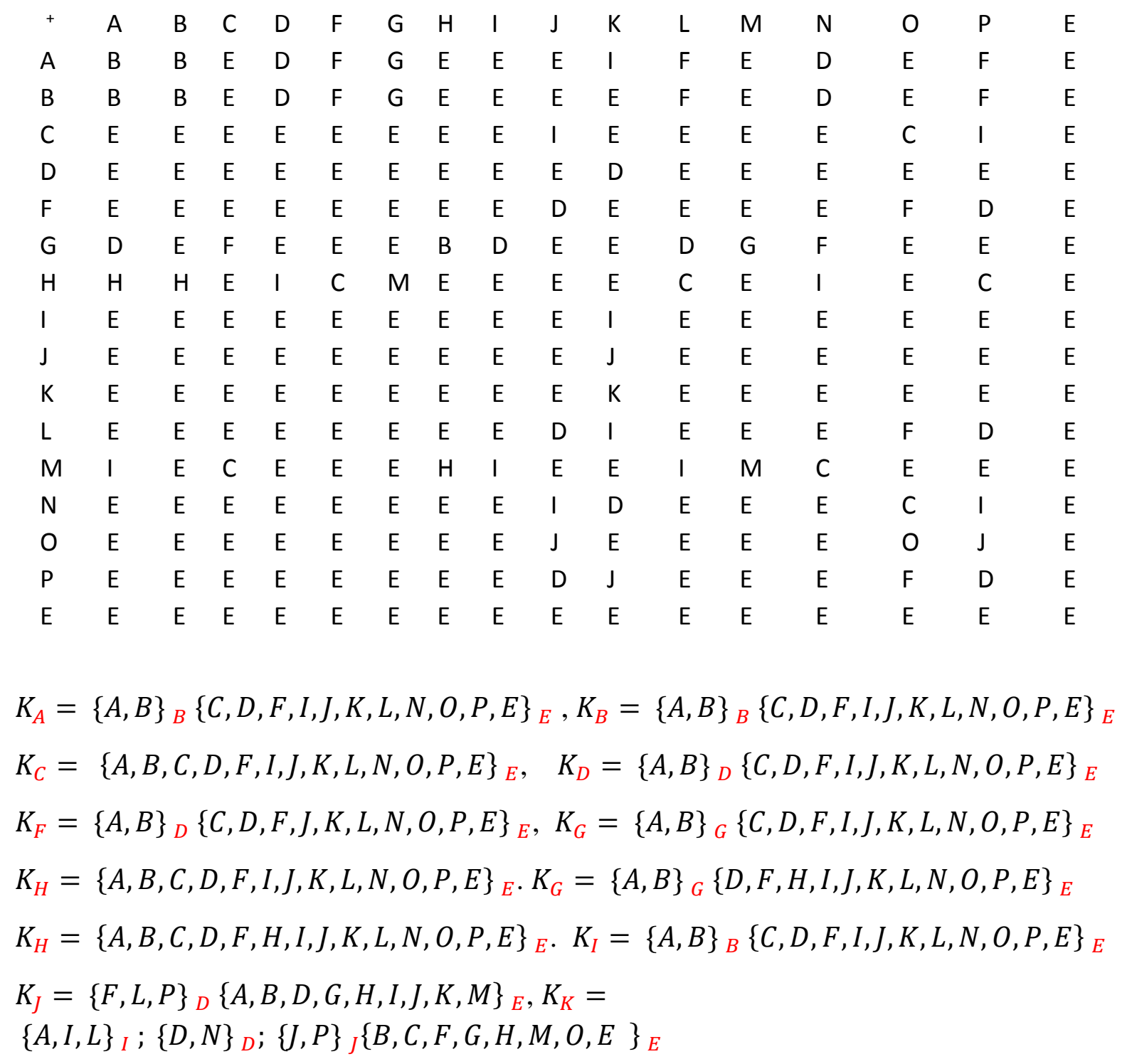




$$
\begin{gathered}
K_{L}=\{A, B\}_{F}\{C, D, F, I, J, K, L, N, O, P, E\}_{E}, K_{M}=\{A, B, C, D, F, H, I J J, K, L, N, O, P, E\}_{E} \\
K_{N}=\{A, B\}_{D}\{C, D, F, I, J, K, L, N, O, P, E\}_{E}, K_{O}=\{F, L, P\}_{F}\{A, B, D, G, H, I, J, K, M, E\}_{E} \\
K_{P}=\{A, B\}_{F}\{D, L\}_{E} ;\{F, L, P\}_{D} ;\{C, N\}_{I}
\end{gathered}
$$

iii. $P T_{\{1,2,3,4\}}$

$$
S_{3}=\{A, B, C, D\}
$$

where $A=\left(\begin{array}{llll}1 & 2 & 3 & 4 \\ 1 & 2 & x & x\end{array}\right), B=\left(\begin{array}{llll}1 & 2 & 3 & 4 \\ x & 2 & x & x\end{array}\right), C=\left(\begin{array}{llll}1 & 2 & 3 & 4 \\ 4 & 2 & x & x\end{array}\right), D=\left(\begin{array}{llll}1 & 2 & 3 & 4 \\ 3 & 2 & x & x\end{array}\right)$

Table $3: \wp T_{\{1,2,3,4,\}}:\left(\begin{array}{llll}1 & 2 & x & x\end{array}\right)$

$\begin{array}{lllll}+ & \mathrm{A} & \mathrm{B} & \mathrm{C} & \mathrm{D} \\ \mathrm{A} & \mathrm{A} & \mathrm{B} & \mathrm{C} & \mathrm{D} \\ \mathrm{B} & \mathrm{B} & \mathrm{B} & \mathrm{B} & \mathrm{B} \\ \mathrm{C} & \mathrm{B} & \mathrm{B} & \mathrm{B} & \mathrm{B} \\ \mathrm{D} & \mathrm{B} & \mathrm{B} & \mathrm{B} & \mathrm{B}\end{array}$

$K_{A}=\{B, C, D\}_{B}, K_{B}=\{A, B, C, D\}_{B}, K_{C}=\{B, C, D\}_{B}, K_{D}=\{B, C, D\}_{B}$

iv. $P T_{\{1,2,3,4,5\}}$

$$
\begin{aligned}
& S_{4}=\{A, B, C, D, E, F, E\} \\
& A=\left(\begin{array}{lllll}
1 & 2 & 3 & 4 & 5 \\
1 & x & x & x & x
\end{array}\right), B=\left(\begin{array}{ccccc}
1 & 2 & 3 & 4 & 5 \\
x & 4 & x & x & x
\end{array}\right), C=\left(\begin{array}{lllll}
1 & 2 & 3 & 4 & 5 \\
5 & x & x & x & x
\end{array}\right), D=\left(\begin{array}{lllll}
1 & 2 & 3 & 4 & 5 \\
4 & x & x & x & x
\end{array}\right), \\
& F=\left(\begin{array}{lllll}
1 & 2 & 3 & 4 & 5 \\
3 & x & x & x & x
\end{array}\right), G=\left(\begin{array}{lllll}
1 & 2 & 3 & 4 & 5 \\
2 & x & x & x & x
\end{array}\right), E=\left(\begin{array}{lllll}
1 & 2 & 3 & 4 & 5 \\
x & x & x & x & x
\end{array}\right) \\
& \text { Table } 4: \wp T_{\{1,2,3,4,5\}}:\left(\begin{array}{lllll}
1 & x & x & x & x
\end{array}\right) \\
& + \text { A B C D F G E }
\end{aligned}
$$

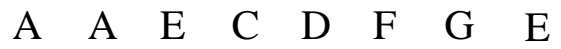

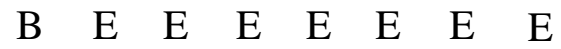

$$
\begin{aligned}
& \begin{array}{llllllll}
C & \mathrm{E} & \mathrm{E} & \mathrm{E} & \mathrm{E} & \mathrm{E} & \mathrm{E} & \mathrm{E}
\end{array} \\
& \begin{array}{llllllll}
\mathrm{D} & \mathrm{E} & \mathrm{E} & \mathrm{E} & \mathrm{E} & \mathrm{E} & \mathrm{E} & \mathrm{E}
\end{array}
\end{aligned}
$$

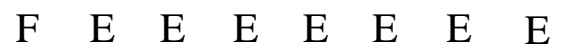

$$
\begin{aligned}
& \begin{array}{llllllll}
G & E & D & E & E & E & E & E
\end{array} \\
& \text { E } \\
& K_{A}=\{B, C, D, F, G, E\}_{E}, K_{B}=\{A, B, C, D, F, E\}_{E}, K_{C}=\{B, C, D, F, G, E\}_{E}, \\
& K_{F}=\{B, C, D, F, G, E\}_{E}, K_{G}=\{B, C, D, F, G, E\}_{E}
\end{aligned}
$$


v. $P T_{\{1,2,3,4,5,6\}}$

$$
\begin{aligned}
& S_{5}=\{A, B, C, D, E, F, G, H, I, J, K, E\} \\
& A=\left(\begin{array}{lllll}
1 & 2 & 3 & 4 & 5 \\
2 & x & x & 5 & 4
\end{array}\right), B=\left(\begin{array}{lllll}
1 & 2 & 3 & 4 & 5 \\
x & x & x & 4 & 5
\end{array}\right), C=\left(\begin{array}{lllll}
1 & 2 & 3 & 4 & 5 \\
x & x & x & 5 & 4
\end{array}\right), D\left(\begin{array}{lllll}
1 & 2 & 3 & 4 & 5 \\
3 & x & x & 4 & 5
\end{array}\right), \\
& F=\left(\begin{array}{lllll}
1 & 2 & 3 & 4 & 5 \\
3 & x & x & 5 & 4
\end{array}\right), G=\left(\begin{array}{lllll}
1 & 2 & 3 & 4 & 5 \\
x & 3 & x & 5 & 4
\end{array}\right), H=\left(\begin{array}{lllll}
1 & 2 & 3 & 4 & 5 \\
x & 2 & x & 4 & 5
\end{array}\right), I=\left(\begin{array}{lllll}
1 & 2 & 3 & 4 & 5 \\
2 & x & x & 4 & 5
\end{array}\right), \\
& J=\left(\begin{array}{lllll}
1 & 2 & 3 & 4 & 5 \\
x & 3 & x & 5 & 4
\end{array}\right), K=\left(\begin{array}{lllll}
1 & 2 & 3 & 4 & 5 \\
x & 2 & x & 4 & 5
\end{array}\right), E=\left(\begin{array}{lllll}
1 & 2 & 3 & 4 & 5 \\
x & x & x & x & x
\end{array}\right)
\end{aligned}
$$

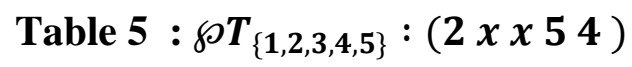

$\begin{array}{llllllllllll}+ & \text { A } & \text { B } & \text { C } & \text { D } & \text { F } & \text { G } & \text { H } & \text { I } & \text { J } & \text { K } & \text { E } \\ \text { A } & \text { B } & \text { C } & \text { B } & \text { C } & \text { B } & \text { F } & \text { I } & \text { C } & \text { D } & \text { A } & \text { E } \\ \text { B } & \text { C } & \text { B } & \text { C } & \text { B } & \text { C } & \text { B } & \text { C } & \text { B } & \text { C } & \text { B } & \text { E } \\ \text { C } & \text { B } & \text { C } & \text { B } & \text { C } & \text { B } & \text { C } & \text { B } & \text { C } & \text { B } & \text { C } & \text { E } \\ \text { D } & \text { C } & \text { B } & \text { C } & \text { B } & \text { C } & \text { B } & \text { C } & \text { B } & \text { C } & \text { B } & \text { E } \\ \text { F } & \text { B } & \text { C } & \text { B } & \text { C } & \text { B } & \text { C } & \text { B } & \text { C } & \text { B } & \text { C } & \text { E } \\ \text { G } & \text { C } & \text { B } & \text { C } & \text { B } & \text { C } & \text { B } & \text { C } & \text { B } & \text { C } & \text { B } & \text { E } \\ \text { H } & \text { B } & \text { C } & \text { B } & \text { C } & \text { B } & \text { J } & \text { K } & \text { C } & \text { G } & \text { H } & \text { E } \\ \text { I } & \text { C } & \text { B } & \text { C } & \text { B } & \text { C } & \text { D } & \text { A } & \text { B } & \text { F } & \text { I } & \text { E } \\ \text { J } & \text { B } & \text { C } & \text { B } & \text { C } & \text { B } & \text { C } & \text { B } & \text { C } & \text { B } & \text { C } & \text { E } \\ \text { K } & \text { C } & \text { B } & \text { C } & \text { B } & \text { C } & \text { G } & \text { H } & \text { B } & \text { J } & \text { K } & \text { E } \\ \text { E } & \text { E } & \text { E } & \text { E } & \text { E } & \text { E } & \text { E } & \text { E } & \text { E } & \text { E } & \text { E } & \text { E }\end{array}$

$$
\begin{aligned}
& K_{A}=\{B, D, G, I, K\}_{C} ;\{A, C, F, H, J\}_{B} ; K_{B}=\{B, D, G, I, K\}_{B} ;\{A, C, F, H, J\}_{C} \\
& K_{C}=\{A, C, F, H, J\}_{B} ;\{B, D, G, I, K\}_{C} ; K_{D}=\{A, C, F, H, J\}_{C} ;\{B, D, G, I, K\}_{B} \\
& K_{F}=\{A, C, F, H, J\}_{B} ;\{B, D, G, I, K\}_{C} K_{G}=\{B, D, G\}_{B} ;\{C, F, J\}_{C} \\
& K_{H}=\{B, D, G\}_{C} ;\{C, F, J\}_{B} ; K_{I}=\{A, C, F, H, J\}_{C} ;\{B, D, G, I, K\}_{B} \\
& K_{J}=\{B, D, G\}_{C} ;\{C, F, J\}_{B} ; K_{K}=\{C, F, J\}_{C} ;\{B, D, G\}_{B} \\
& K_{E}=\{A, B, C, D, E, F, G, H, I, J, K, E\}_{E}
\end{aligned}
$$

vi. $P T_{\{1,2,3,4,5,6\}}$

$$
\begin{aligned}
& S_{6}=\{A, B, C, D, E\} \\
A & =\left(\begin{array}{llllll}
1 & 2 & 3 & 4 & 5 & 6 \\
2 & 4 & 3 & x & 5 & 1
\end{array}\right), B=\left(\begin{array}{llllll}
1 & 2 & 3 & 4 & 5 & 6 \\
4 & x & 3 & x & 5 & 2
\end{array}\right), C=\left(\begin{array}{llllll}
1 & 2 & 3 & 4 & 5 & 6 \\
x & x & 3 & x & 5 & 4
\end{array}\right), \\
D & =\left(\begin{array}{llllll}
1 & 2 & 3 & 4 & 5 & 6 \\
x & x & 3 & x & 5 & x
\end{array}\right), \quad E=\left(\begin{array}{llllll}
1 & 2 & 3 & 4 & 5 & 6 \\
x & x & x & x & x & x
\end{array}\right)
\end{aligned}
$$




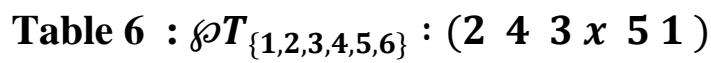

$\begin{array}{llllll}+ & \mathrm{A} & \mathrm{B} & \mathrm{C} & \mathrm{D} & \mathrm{E} \\ \mathrm{A} & \mathrm{B} & \mathrm{C} & \mathrm{D} & \mathrm{D} & \mathrm{E} \\ \mathrm{B} & \mathrm{C} & \mathrm{D} & \mathrm{D} & \mathrm{D} & \mathrm{E} \\ \mathrm{C} & \mathrm{D} & \mathrm{D} & \mathrm{C} & \mathrm{D} & \mathrm{E} \\ \mathrm{D} & \mathrm{D} & \mathrm{D} & \mathrm{D} & \mathrm{D} & \mathrm{E} \\ \mathrm{E} & \mathrm{E} & \mathrm{E} & \mathrm{E} & \mathrm{E} & \mathrm{E}\end{array}$

$K_{A}=\{C, D\}_{D} ; K_{B}=\{B, C, D\}_{B} ; K_{C}=\{A, B, D\}_{D}$

$K_{D}=\{A, B, C, D\}_{D} ; K_{E}=\{A, B, C, D, E\}_{E}$

vii. $P T_{\{1,2,3,4,5,6,7\}}$

$S_{7}=\{A, B, C, D, E, F, G, H, I, J, K, E\}$

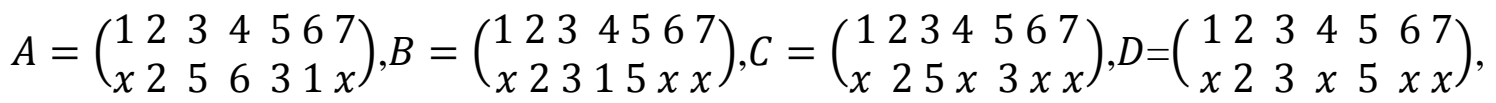

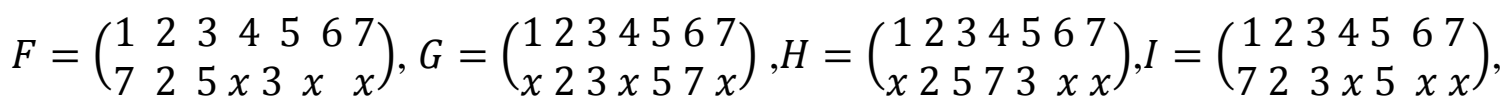

$J=\left(\begin{array}{lllllll}1 & 2 & 3 & 4 & 5 & 6 & 7 \\ x & 2 & 5 & x & 3 & 7 & x\end{array}\right), K=\left(\begin{array}{lllllll}1 & 2 & 3 & 4 & 5 & 6 & 7 \\ x & 2 & 3 & 7 & 5 & x & x\end{array}\right), E=\left(\begin{array}{lllllll}1 & 2 & 3 & 4 & 5 & 6 & 7 \\ x & x & x & x & x & x & x\end{array}\right)$

Table $7: \wp T_{\{1,2,3,4,5,6,7\}}:\left(\begin{array}{lll}x & 25631 x\end{array}\right)$

$\begin{array}{llllllllllll}+ & \text { A } & \text { B } & \text { C } & \text { D } & \text { F } & \text { G } & \text { H } & \text { I } & \text { J } & \text { K } & \text { E } \\ \text { A } & \text { B } & \text { C } & \text { D } & \text { C } & \text { G } & \text { H } & \text { D } & \text { J } & \text { K } & \text { C } & \text { E } \\ \text { B } & \text { C } & \text { D } & \text { C } & \text { D } & \text { H } & \text { D } & \text { C } & \text { K } & \text { C } & \text { D } & \text { E } \\ \text { C } & \text { D } & \text { C } & \text { D } & \text { C } & \text { D } & \text { C } & \text { D } & \text { C } & \text { D } & \text { C } & \text { E } \\ \text { D } & \text { C } & \text { D } & \text { C } & \text { D } & \text { C } & \text { D } & \text { C } & \text { D } & \text { C } & \text { D } & \text { E } \\ \text { F } & \text { D } & \text { C } & \text { D } & \text { C } & \text { D } & \text { C } & \text { D } & \text { C } & \text { D } & \text { C } & \text { E } \\ \text { G } & \text { C } & \text { D } & \text { C } & \text { D } & \text { C } & \text { D } & \text { C } & \text { D } & \text { C } & \text { D } & \text { E } \\ \text { H } & \text { D } & \text { C } & \text { D } & \text { C } & \text { D } & \text { C } & \text { D } & \text { C } & \text { D } & \text { C } & \text { E } \\ \text { I } & \text { C } & \text { D } & \text { C } & \text { D } & \text { C } & \text { D } & \text { C } & \text { D } & \text { C } & \text { D } & \text { E } \\ \text { J } & \text { D } & \text { C } & \text { D } & \text { C } & \text { D } & \text { C } & \text { D } & \text { C } & \text { D } & \text { C } & \text { E } \\ \text { K } & \text { C } & \text { D } & \text { C } & \text { D } & \text { C } & \text { D } & \text { C } & \text { D } & \text { C } & \text { D } & \text { E } \\ \text { E } & \text { E } & \text { E } & \text { E } & \text { E } & \text { E } & \text { E } & \text { E } & \text { E } & \text { E } & \text { E } & \text { E }\end{array}$

$K_{A}=\{C, F, H, J\}_{D} ;\{B, D, G, I, K\}_{C}, K_{B}=\{A, C, F, H, J\}_{C} ;\{B, D, G, I, K\}_{D}$

$K_{C}=\{A, C, F, H, J\}_{D} ;\{B, D, G, I, K\}_{C}, K_{D}=\{A, C, F, H, J\}_{C} ;\{B, D, G, I, K\}_{D}$

$K_{F}=\{C, F, H, J\}_{D} ;\{D, G, I, K\}_{C}, K_{G}=\{C, F, H, J\}_{C} ;\{B, D, G, I, K\}_{D}$ 


$$
\begin{aligned}
& K_{H}=\{A, C, F, H, J\}_{D} ;\{B, D, G, I, K\}_{C}, K_{I}=\{C, F, H, J\}_{C} ;\{D, G, I, K\}_{D} \\
& K_{J}=\{C, F, H, J\}_{D} ;\{B, D, G, I, K\}_{C}, K_{K}=\{A, C, F, H, J\}_{C} ;\{B, D, G, I, K\}_{D} \\
& K_{E}=\{A, B, C, D, F, G, H, I, J, K, E\}_{E}
\end{aligned}
$$

viii. $P T_{\{1,2,3,4,5,6,7,8\}}$

$$
\begin{aligned}
& S_{8}=\{A, B, C, D, F, G, H, E\}
\end{aligned}
$$

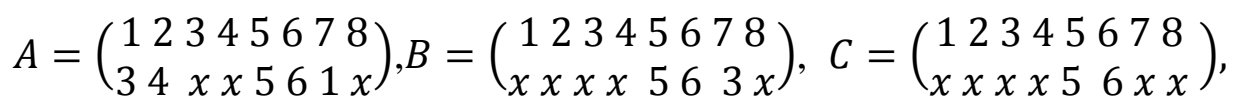

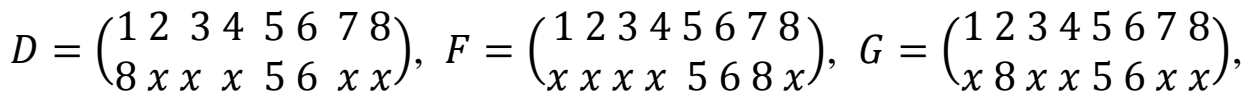

$$
\begin{aligned}
& H=\left(\begin{array}{llllllll}
1 & 2 & 3 & 4 & 5 & 6 & 7 & 8 \\
x & 8 & x & x & 5 & 6 & x & x
\end{array}\right), E=\left(\begin{array}{llllllll}
1 & 2 & 3 & 4 & 5 & 6 & 7 & 8 \\
x & x & x & x & x & x & x & x
\end{array}\right)
\end{aligned}
$$

Table $8: \wp T_{\{1,2,3,4,5,6,7,8\}}:(34 x x 561 x)$

$\begin{array}{lllllllll}+ & \mathrm{A} & \mathrm{B} & \mathrm{C} & \mathrm{D} & \mathrm{F} & \mathrm{G} & \mathrm{H} & \mathrm{E} \\ \mathrm{A} & \mathrm{B} & \mathrm{C} & \mathrm{C} & \mathrm{F} & \mathrm{C} & \mathrm{C} & \mathrm{D} & \mathrm{E} \\ \mathrm{B} & \mathrm{C} & \mathrm{C} & \mathrm{C} & \mathrm{C} & \mathrm{C} & \mathrm{C} & \mathrm{F} & \mathrm{E} \\ \mathrm{C} & \mathrm{C} & \mathrm{C} & \mathrm{C} & \mathrm{C} & \mathrm{C} & \mathrm{C} & \mathrm{C} & \mathrm{E} \\ \mathrm{D} & \mathrm{C} & \mathrm{C} & \mathrm{C} & \mathrm{C} & \mathrm{C} & \mathrm{C} & \mathrm{C} & \mathrm{E} \\ \mathrm{F} & \mathrm{C} & \mathrm{C} & \mathrm{C} & \mathrm{C} & \mathrm{C} & \mathrm{C} & \mathrm{C} & \mathrm{E} \\ \mathrm{G} & \mathrm{C} & \mathrm{C} & \mathrm{C} & \mathrm{C} & \mathrm{C} & \mathrm{C} & \mathrm{C} & \mathrm{E} \\ \mathrm{H} & \mathrm{C} & \mathrm{C} & \mathrm{C} & \mathrm{C} & \mathrm{C} & \mathrm{C} & \mathrm{C} & \mathrm{E} \\ \mathrm{E} & \mathrm{E} & \mathrm{E} & \mathrm{E} & \mathrm{E} & \mathrm{E} & \mathrm{E} & \mathrm{E} & \mathrm{E}\end{array}$

$$
\begin{aligned}
& K_{A}=\{B, C, D, F, G, H\}_{C}, K_{B}=\{A, B, C, D, F, G, H\}_{C} ; K_{C}=\{A, B, C, D, F, G, H\}_{C} \\
& K_{D}=\{B, C, D, F, G, H\}_{C} ; K_{F}=\{A, B, C, D, F, G, H\}_{C}, K_{G}=\{A, B, C, D, F, G, H\}_{C} \\
& K_{H}=\{A, B, C, D, F, G, H\}_{C} ; K_{E}=\{A, B, C, D, F, G, H, E\}_{E}
\end{aligned}
$$

ix. $P T_{\{1,2,3,4,5,6,7,8,9\}}$

$$
\begin{aligned}
& S_{9}=\{A, B, C, D, F, G, H, I, J, E\}
\end{aligned}
$$

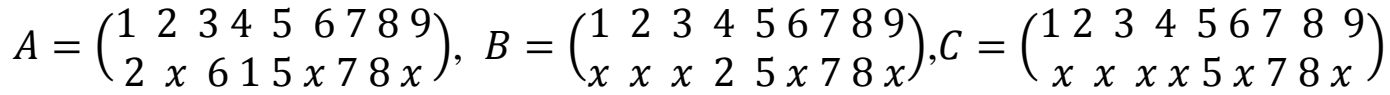

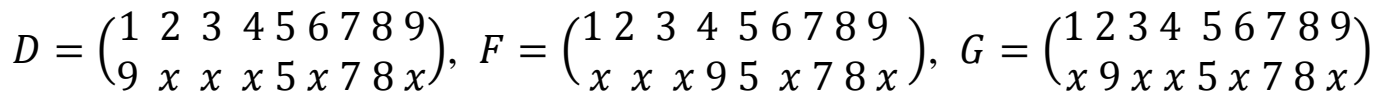




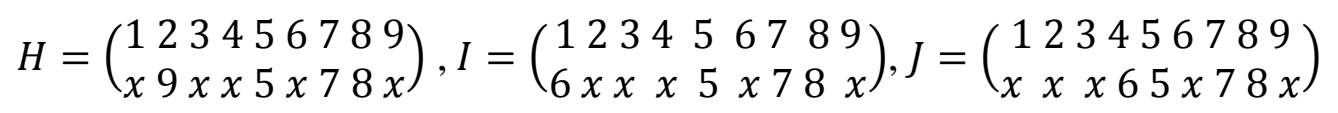

Table $9: \wp T_{\{1,2,3,4,5,6,7,8,9\}}:\left(\begin{array}{l}2 x 615 x 78 x) \\ x\end{array}\right.$

$$
\begin{aligned}
& \begin{array}{llllllllll}
+ & \mathrm{A} & \mathrm{B} & \mathrm{C} & \mathrm{D} & \mathrm{F} & \mathrm{G} & \mathrm{H} & \mathrm{I} & \mathrm{J}
\end{array} \\
& \begin{array}{llllllllll}
\mathrm{A} & \mathrm{B} & \mathrm{C} & \mathrm{C} & \mathrm{F} & \mathrm{C} & \mathrm{D} & \mathrm{I} & \mathrm{C} & \mathrm{C}
\end{array} \\
& \begin{array}{llllllllll}
\mathrm{B} & \mathrm{C} & \mathrm{C} & \mathrm{C} & \mathrm{C} & \mathrm{C} & \mathrm{F} & \mathrm{C} & \mathrm{C} & \mathrm{C}
\end{array} \\
& \begin{array}{llllllllll}
\mathrm{C} & \mathrm{C} & \mathrm{C} & \mathrm{C} & \mathrm{C} & \mathrm{C} & \mathrm{C} & \mathrm{C} & \mathrm{C} & \mathrm{C}
\end{array} \\
& \begin{array}{llllllllllll}
\mathrm{D} & \mathrm{C} & \mathrm{C} & \mathrm{C} & \mathrm{C} & \mathrm{C} & \mathrm{C} & \mathrm{C} & \mathrm{C} & \mathrm{C}
\end{array} \\
& \begin{array}{llllllllll}
\mathrm{F} & \mathrm{C} & \mathrm{C} & \mathrm{C} & \mathrm{C} & \mathrm{C} & \mathrm{C} & \mathrm{C} & \mathrm{C} & \mathrm{C}
\end{array}
\end{aligned}
$$

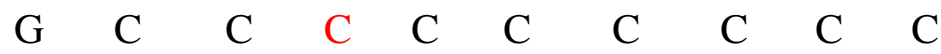

$$
\begin{aligned}
& \begin{array}{llllllllll}
\mathrm{H} & \mathrm{C} & \mathrm{C} & \mathrm{C} & \mathrm{C} & \mathrm{C} & \mathrm{C} & \mathrm{C} & \mathrm{C} & \mathrm{C}
\end{array} \\
& \begin{array}{llllllllll}
\mathrm{I} & \mathrm{C} & \mathrm{C} & \mathrm{C} & \mathrm{C} & \mathrm{C} & \mathrm{C} & \mathrm{C} & \mathrm{C} & \mathrm{C}
\end{array}
\end{aligned}
$$

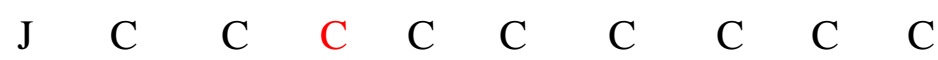

$$
\begin{aligned}
& K_{A}=\{B, C, D, F, G, H, I, J\}_{C}: K_{B}=\{A, B, C, D, F, G, H, I, J, E\}_{C}: \\
& K_{C}=\{A, B, C, D, F, G, H, I, J, E\}_{C} \\
& K_{D}=\{B, C, D, F, G, H, I, J\}_{C} ; K_{F}=\{A, B, C, D, F, G, H, I, J\}_{C} ; K_{G}=\{C, D, F, G, H, I, J\}_{C} \\
& K_{H}=\{B, C, D, F, G, H, I, J\}_{C} ; K_{I}=\{A, B, C, D, F, G, H, I, J\}_{C} ; \\
& K_{J}=\{A, B, C, D, F, G, H, I, J\}_{C}
\end{aligned}
$$

$x . P T_{\{1,2,3,4,5,6,7,8,9,10\}}$

$$
\begin{aligned}
& S_{10}=\{A, B, C, D, F, G, H, I, J, K, L, M, N, E\}
\end{aligned}
$$

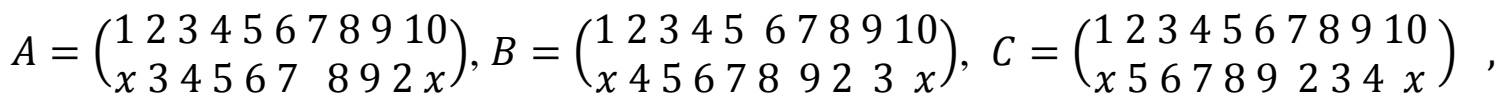

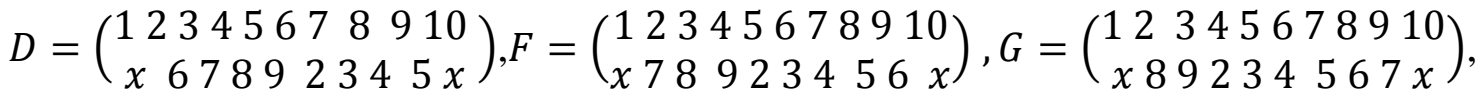

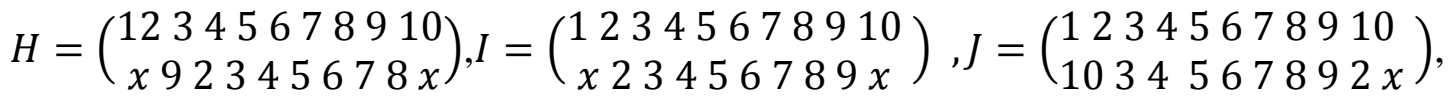

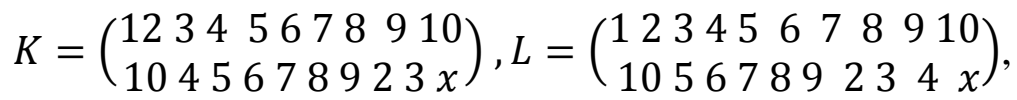

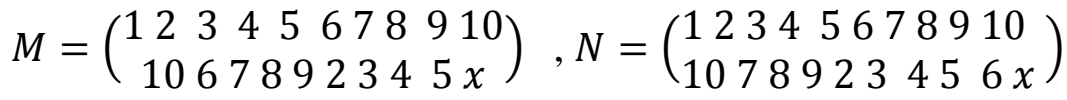


Table 10: $\wp T_{\{1,2,3,4,5,6,7,8,9,10\}}:(x 34567892 x)$

$\begin{array}{llllllllllllll}+ & \text { A } & \text { B } & \text { C } & \text { D } & \text { F } & \text { G } & \text { H } & \text { I } & \text { J } & \text { K } & \text { L } & \text { M } & \text { N } \\ \text { A } & \text { B } & \text { C } & \text { D } & \text { F } & \text { G } & \text { H } & \text { I } & \text { A } & \text { B } & \text { C } & \text { D } & \text { F } & \text { G } \\ \text { B } & \text { C } & \text { D } & \text { F } & \text { G } & \text { H } & \text { I } & \text { A } & \text { B } & \text { C } & \text { D } & \text { F } & \text { G } & \text { H } \\ \text { C } & \text { D } & \text { F } & \text { G } & \text { H } & \text { I } & \text { A } & \text { B } & \text { C } & \text { D } & \text { F } & \text { G } & \text { H } & \text { I } \\ \text { D } & \text { F } & \text { G } & \text { H } & \text { I } & \text { A } & \text { B } & \text { C } & \text { D } & \text { F } & \text { G } & \text { H } & \text { I } & \text { A } \\ \text { F } & \text { G } & \text { H } & \text { I } & \text { A } & \text { B } & \text { C } & \text { D } & \text { F } & \text { G } & \text { H } & \text { I } & \text { A } & \text { B } \\ \text { G } & \text { H } & \text { I } & \text { A } & \text { B } & \text { C } & \text { D } & \text { F } & \text { G } & \text { H } & \text { I } & \text { A } & \text { B } & \text { C } \\ \text { H } & \text { I } & \text { A } & \text { B } & \text { C } & \text { D } & \text { F } & \text { G } & \text { H } & \text { I } & \text { A } & \text { B } & \text { C } & \text { D } \\ \text { I } & \text { A } & \text { B } & \text { C } & \text { D } & \text { F } & \text { G } & \text { H } & \text { I } & \text { A } & \text { B } & \text { C } & \text { D } & \text { F } \\ \text { J } & \text { B } & \text { C } & \text { D } & \text { F } & \text { G } & \text { H } & \text { I } & \text { A } & \text { B } & \text { C } & \text { D } & \text { F } & \text { G } \\ \text { K } & \text { C } & \text { D } & \text { F } & \text { G } & \text { H } & \text { I } & \text { A } & \text { B } & \text { C } & \text { D } & \text { F } & \text { G } & \text { H } \\ \text { L } & \text { D } & \text { F } & \text { G } & \text { H } & \text { I } & \text { A } & \text { B } & \text { C } & \text { D } & \text { F } & \text { G } & \text { H } & \text { I } \\ \text { M } & \text { F } & \text { G } & \text { H } & \text { I } & \text { A } & \text { B } & \text { C } & \text { D } & \text { F } & \text { G } & \text { H } & \text { I } & \text { A } \\ \text { N } & \text { G } & \text { H } & \text { I } & \text { A } & \text { B } & \text { C } & \text { D } & \text { F } & \text { G } & \text { H } & \text { I } & \text { A } & \text { B }\end{array}$

$K_{A}=\{A, J\}_{B} ;\{B, K\}_{C} ;\{C, L\}_{D} ;\{F, N\}_{G} ;\{D, M\}_{F}$

$K_{B}=\{A, J\}_{C} ;\{B, K\}_{D} ;\{C, L\}_{F} ;\{F, N\}_{H} ;\{D, M\}_{G}$

$K_{C}=\{A, J\}_{D} ;\{B, K\}_{F} ;\{C, L\}_{G} ;\{F, N\}_{I} ;\{D, M\}_{H}$

$K_{D}=\{A, J\}_{F} ;\{B, K\}_{G} ;\{C, L\}_{H} ;\{F, N\}_{A} ;\{D, M\}_{I}$

$K_{F}=\{A, J\}_{F} ;\{B, K\}_{H} ;\{C, L\}_{I} ;\{F, N\}_{B} ;\{D, M\}_{A}$

$K_{G}=\{A, J\}_{H} ;\{B, K\}_{I} ;\{C, L\}_{A} ;\{F, N\}_{C} ;\{D, M\}_{B}$

$K_{H}=\{A, J\}_{I} ;\{B, K\}_{A} ;\{C, L\}_{B} ;\{F, N\}_{D} ;\{D, M\}_{C}$

$K_{I}=\{A, J\}_{A} ;\{B, K\}_{B} ;\{C, L\}_{C} ;\{F, N\}_{F} ;\{D, M\}_{D}$

$K_{J}=\{A, J\}_{B} ;\{B, K\}_{C} ;\{C, L\}_{D} ;\{F, N\}_{G} ;\{D, M\}_{F}$

$K_{K}=\{A, J\}_{C} ;\{B, K\}_{D} ;\{C, L\}_{F} ;\{F, N\}_{H} ;\{D, M\}_{G}$

$K_{L}=\{A, J\}_{D} ;\{B, K\}_{F} ;\{C, L\}_{G} ;\{F, N\}_{I} ;\{D, M\}_{H}$

$K_{M}=\{A, J\}_{F} ;\{B, K\}_{G} ;\{C, L\}_{H} ;\{F, N\}_{A} ;\{D, M\}_{I}$

$K_{N}=\{A, J\}_{G} ;\{B, K\}_{H} ;\{C, L\}_{I} ;\{F, N\}_{B} ;\{D, M\}_{A}$ 


\section{APPLICATION}

Applications of partial transformations include : Rail track lining ( in exchange programme ), traffic flow algorithms, fluid mechanics, movement algorithm and artificial intelligence for digitalized change to create intriguing patterns. As kernel is a process manager, a foundational layer of operating system, managing hardware resources like RAM and CPU and applications like running processes managing hard disks and interrupts are processed faster with the kernels. The kernels computed form a whole new foundational layer of operating systems.

\section{CONCLUSION}

This work extends Abubakar et.al. (2020) where the congruence classes $\widetilde{R_{S}}$ and the semilattices of idempotents $\widetilde{E_{S}}$ of some left restriction semigroups were computed, here the kernels of the left restriction semigroups were computed and enumerated.

\section{REFERENCES:}

[1] R. B. Abubakar; Asibong-ibe, U. E. \& Jackreece, P. C.; "Computing the Congruence class of some left restriction semigroups in $\wp \widetilde{\Xi}_{X}^{\prime}$ : Journal of semigroup theory and applications (2020) article ID 2 accessible at https://doi.org/10.28919/jsta/4378

[2] C. Hollings; "From right PP monoids to restriction semigroups: A survey" : European Journal of pure and appl maths, 2 (1), 21-57, (2009)

[3] C. Hollings, \& Gould, V. ; Restriction semigroups and inductive constellations. Comm Algebra ,38, 261-287 (2010)

[4] J. Howie ; Fundamentals of semigroup theory. Oxford university press, New York (2003)

[5] A. O. Kuku ; Abstract Algebra, Ibadan University Press, Ibadan (1992)

[6] E. Manes \& Amherst, U.; Partial Transformations: Semigroups, Categories and equations. Semigroups/Categories workshop University of Ottawa (2010)

[7] M. Petrich \& Rankin, S. ;The kernel-Trace approach to right congruences on an inverse Semigroup . Transactions of the American Mathematical Society, 330(2), 917-920, (1992) 